\title{
GROWTH-INDUCED BIREFRINGENCE IN LPE-GROWN IRON GARNET FILMS
}

\author{
N. KOSHIZUKA, K. ANDO, and T. OKUDA \\ Electrotechnical Laboratory, Sakura-mura, Ibaraki 305, Japan
}

\section{ABSTRACT}

The optical birefringence of various LPE-grown rare-earth and Bi-substituted iron garnet films are studied. In addition to the stress-induced birefringence, it is shown that there exists growthinduced birefringence $\Delta n_{f}{ }_{G}$ in the films containing light rare-earth or $B i$ ions. In $B i$ garnet films the $\Delta n_{f}$ disappears on annealing and the thermal behavior of $\Delta n_{f}$ is similar to that of the growth-induced uniaxial magnetic anisotropy constant $\mathrm{K}_{\mathrm{U}} \mathrm{G}$. Furthermore, the $\mathrm{Bi}$ content dependence of $\Delta n_{f} G^{G}$ is similar to that of $K_{u}{ }^{G}$. As a result, it is suggested that the $\Delta n_{f} G$ and $K_{u}{ }^{G}$ originate in the same microscopic structure which is of an anisotropic character. It is pointed out that the positive sign and the magnitude of $\Delta n_{f}{ }^{G}$ in $B i$ garnet films are effective for the phase matching between TE and TM modes in a planar waveguide.

\section{INTRODUCTION}

Waveguide type optical devices are expected to play important roles in future optical communication and information processing systems. As for magneto-optical devices, nonreciprocal devices such as isolators and circulators are indispensable to prevent unstable laser oscillation due to the backward beam and to split the forward and backward beans [1].

Rare-earth iron garnet films are thought to be most promising materials because of their high magneto-optical constants, optically isotropic crystal structure and a variety of magnetic properties. Especially Bi-substituted iron garnet films are the materials of great interest [2,3]. The enhancement of Faraday rotation and the increase in refractive index and the Curie temperature occur with $\mathrm{Bi}$ substitution. Furthermore, it is known that the uniaxial magnetic anisotropy is induced in LPE-grown Bi garnet films. These studies have been extensively done by many groups, in particular, by Hansen et al. of Philips Research Laboratories [3].

Since the refractive index of these films is higher than that of the substrates such as GGG, these films are also suitable as waveguide materials. However, there remain several basic problems such as phase matching between TE and TM modes and microfabrication techniques of garnet films. As for the phase matching, it is known that the film birefringence $\Delta n_{f}\left(=n_{T M}-n_{T E}\right)$ is effective to compensate for the phase mismatch due to the planar structure [4]. Furthermore, the magnitude of $\Delta n_{f}$ can be controlled by the stress-induced birefringence $\Delta n_{f}$ caused by the lattice mismatch between film and substrate [4 6]. In view of these considerations, studies on the birefringence of various rare-earth and Bi-substituted iron garnet films were carried out $17 \sim 141$. As a result, we

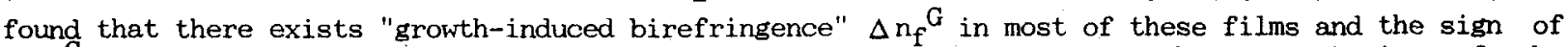
$\Delta n_{f}$ is positive in Bi-substituted iron garnet films. This paper describes the relations of the optical birefringence with the lattice mismatch, annealing treatment, and Bi content in LPE-grown garnet films.

\section{MODE CONVERSION AND BIREFRINGENCE}

So far, many theoretical and experimental studies on waveguide type non-reciprocal devices or switches have been made in the last decade [1,15 24]. In these devices magneto-optical power conversion between TE (transverse electric) and IM (transverse magnetic) modes plays a crucial role. The power conversion between TE and TM modes in the Faraday configuration is expressed by the equation [17]

$$
R(L)=\frac{K^{2}}{K^{2}+(\Delta \beta / 2)^{2}} \sin ^{2}\left(\sqrt{K^{2}+(\Delta \beta / 2)^{2}} \cdot L\right),
$$

where $\mathrm{K}$ is the coupling constant which equals the Faraday rotation coefficient, $\Delta \beta$ is the difference between the propagation constants for the two modes $(\Delta \beta=\beta \mathrm{TM}-\beta \mathrm{TE})$, and $\mathrm{L}$ is the propagation length. From eq.(1), it turns out that complete mode conversion occurs under the phasematching condition, i.e. $\Delta \beta=0$.

The effective refractive indices for $\mathrm{TE}$ and $\mathrm{TM}$ modes are defined by $N_{\mathrm{TE}}=\beta \mathrm{TE} / \mathrm{k}$ and $N_{\mathrm{TM}}=\beta \mathrm{TM} / \mathrm{k}$, where $k$ is the propagation constant of light in vacuum $(k=2 \pi / \lambda)$, and they have different dispersion curves to the film thickness. In an optically isotropic waveguide, $\mathrm{N}_{\mathrm{TM}}\left\langle\mathrm{N}_{\mathrm{TE}}\right.$, i.e. $\Delta \beta_{i s o}<0$, because the phase changes suffered by the beam at the film-substrate and film-air interfaces are different between the TE and TM waves, and in general the velocity of the TM wave is slightly faster than that of the TE wave. The magnitude of $\Delta \beta$ iso can be calculated from the 
values of film thickness $d$, refractive indices of film $n_{f}$, substrate $n_{s}$ and air $n_{a}$. On the other hand, in an anisotropic case, the degeneracy of the effective refractive indices is expected under a certain condition. In a film with the birefringence of $\Delta n_{f}\left(=n_{T M} n_{T E}\right)$, the phase difference is expressed to a good approximation by

$$
\Delta \beta=\Delta \beta \text { iso }+\mathrm{k} \Delta \mathrm{n}_{\mathrm{f}}
$$

Here, the phase matching condition $\Delta \beta=0$ is satisfied if $\Delta n_{\mathrm{f}}=-\Delta \beta_{\text {iso }} / \mathrm{k}$. Since the sign of $\Delta \beta_{\text {iso }}$ is negative, it is clear that the $\Delta n_{f}$ with positive sign and proper magnitude is necessary for $\triangle \beta=0$.

\section{EXPERTMENTAL}

Films of rare-earth and $\mathrm{Bi}$-substituted iron garnets were grown on (111)-oriented pure and CaMgZr-substituted $\mathrm{Gd}_{3} \mathrm{Ga}_{5} \mathrm{O}_{12}$ (GGG) substrates by liquid-phase epitaxy (LPE). A $\mathrm{PbO}-\mathrm{B}_{2} \mathrm{O}_{3}$ or $\mathrm{PbO}$ $\mathrm{B}_{2} \mathrm{O}_{3}-\mathrm{Bi}_{2} \mathrm{O}_{3}$ flux was used as a solvent. The compositions were determined mainly by means of electron probe $X$-ray microanalyser. The lattice mismatch perpendicular to the film surface $\Delta a^{\perp}\left(=a_{s}-a_{f}{ }^{\perp}\right)$ was measured by X-ray double-crystal diffraction method. The $a_{s}$ and $a_{f}{ }^{1}$ are the lattice parameters of the substrate and film perpendicular to the surface, respectively.

Waveguiding experiments were performed using a $1.15 \mu \mathrm{m}$ He-Ne laser beam and two prisms for coupling of the laser beam. The film thickness $d$ and the refractive index $n_{f}$ were determined by analyzing the mode coupling angles. Power conversion efficiencies $R(L)$ were measured by applying magnetic field parallel to the film plane. The values of $K$ and $\Delta \beta$ were determined by analyzing the propagation-length dependence of $R(L)$. From eq. (2), we obtain the value of $\Delta n_{f}$ using the calculated value of $\Delta \beta_{\text {iso. }}$ Stress-induced birefringence $\Delta n_{f}$ is estimated from the following relation:

$$
\Delta n_{f}^{S}=2 B_{[111]^{C}}{ }_{44} \Delta a^{\perp} / a_{f}
$$

where $B_{[111]}$ is the stress-optical constant for stress in the [111] direction and $C_{44}$ the elastic constant. Values of $\mathrm{B}_{[111]}\left(=3 \times 10^{-13} \mathrm{~cm}^{2} / \mathrm{dyn}\right)$ and $\mathrm{C}_{44}\left(=7.65 \times 10^{11} \mathrm{dyn} / \mathrm{cm}^{2}\right)$ of YIG were adopted in the calculation. The saturation magnetization $4 \pi \mathrm{Ms}$ was measured by using a vibrating sample magnetometer (VSM). The uniaxial magnetic anisotropy constants were determind by analyzing the ferromagnetic resonance (FMR) spectra. The Faraday rotation was measured by using a polarization modulation type Faraday rotation spectrometer (Jasco MOE-7).

\section{RESULTS AND DISCUSSION}

4-1 Dependence of birefringence on lattice mismatch

We first studied the dependence of $\Delta n_{f}$ on lattice mismatch in YNdIG films which have in-plane magnetization [7]. Fig.1 shows the experimental values of $\Delta n_{f}$ and the calculated $\Delta n_{f}{ }^{S}$ as a function of growth temperature $\mathrm{Tg}$, where the saturation temperature of the melt was $1013^{\circ} \mathrm{C}$. One can see here that there exists a negative gap $\left(\Delta n_{f}-\Delta n_{f}\langle 0)\right.$ between $\Delta n_{f}$ and $\Delta n_{f}$ even in misfit-free films $\left(\Delta a^{\perp}=0\right)$. However, for YIG films which were prepared under the similar condition, such a difference was negligibly small.

Lattice mismatch dependence of $\Delta n_{f}$ was studied for other systens [9]. Figs.2 and 3 show the $\Delta n_{f}$ and $\Delta n_{f} S$ as a function of $\Delta a^{\perp}$ in YSmIG and YGdIG films, respectively. In these cases, $\rightarrow$ we also find that $\Delta n_{f}<\Delta n_{f}$.

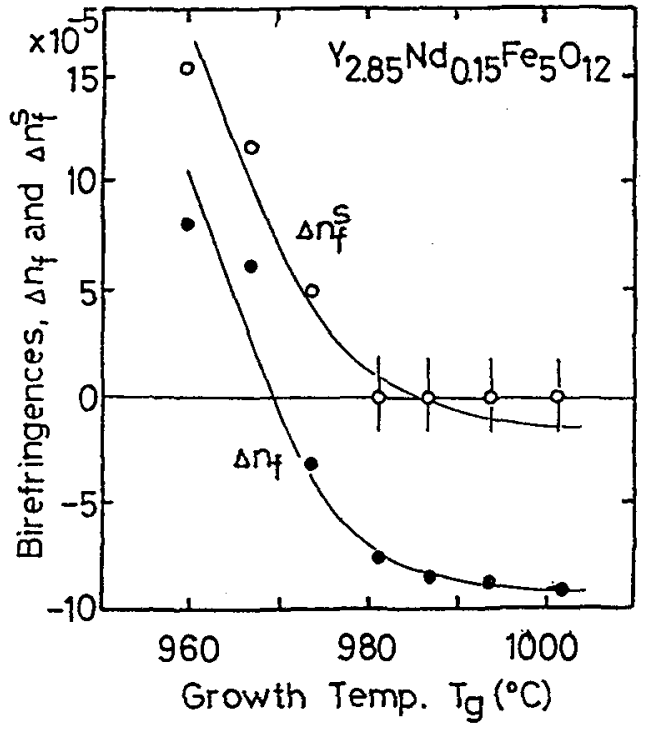

Fig.1 Film birefringence $\Delta \mathrm{n}_{\mathrm{f}}$ and stress-induced birefringence $\Delta n_{f}$ in YNaIG films as a function of growth temperature [9].

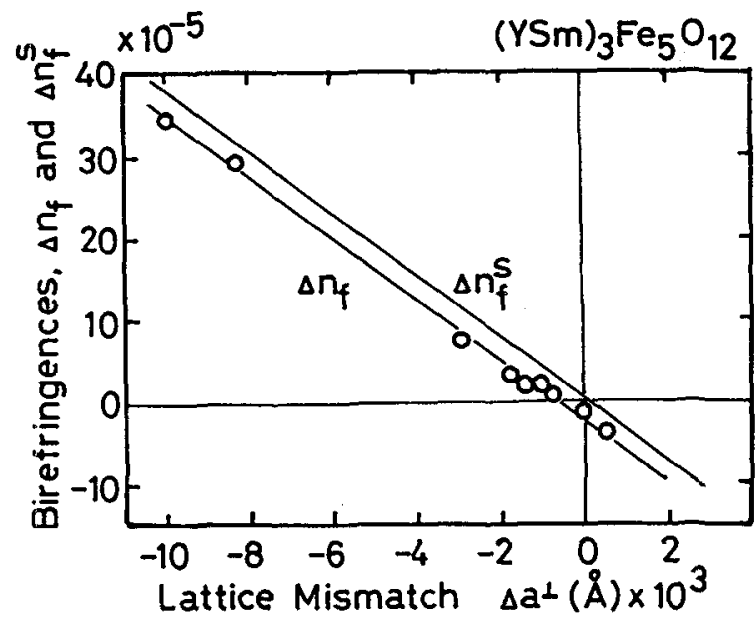

Fig.2 Film birefringence $\Delta \mathrm{n}_{f}$ and stress-induced birefringence $\Delta n_{f}$ in YSmIG films as a function of lattice mismatch $\Delta \mathbf{a}^{\perp}[9]$. 
Table 1 Physical and guided-wave properties of BiPrYbGaIG films at $\lambda=1.15 \mu \mathrm{m}[10]$. $\mathrm{Tg}:$ growth temperature, $\mathrm{d}:$ film thickness, $\Delta a \perp$ : lattice mismatch, $n_{f}:$ refractive index of film, $\alpha$ : absorption loss, $K$ : coupling constant, $\Delta \beta$ : phase mismatch between $T E_{0}$ and $T M_{0}$ modes, $\Delta n_{f}:$ film birefringence, $\Delta n_{\mathrm{f}} \mathrm{s}$ : stress-induced birefringence, $4 \pi$ Ms : saturation magnetization.

\begin{tabular}{|c|c|c|c|c|c|c|c|c|c|c|}
\hline Material No. & $\begin{array}{l}T_{5} \\
\left.{ }^{\circ} \mathrm{C}\right)\end{array}$ & $\underset{(\mu \mathrm{m})}{d}$ & $\begin{array}{l}\Delta a^{2} \\
(A)\end{array}$ & $\mathrm{n}_{\mathrm{f}}$ & $\underset{(\mathrm{dB} / \mathrm{cm})}{\alpha}$ & $\underset{(\mathrm{rad} / \mathrm{cm})}{K}$ & $\begin{array}{c}\Delta \beta \\
(\mathrm{rad} / \mathrm{cm})\end{array}$ & $\Delta n_{f}$ & $\Delta n_{f} S$ & $\begin{array}{c}4 \pi M_{S} \\
\text { (G) }\end{array}$ \\
\hline & & & $10^{-s}$ & & & & & $10^{-4}$ & $10^{-4}$ & \\
\hline 62 & 810 & 3.92 & 7.8 & 2.225 & 14 & -12.6 & -20 & 0.2 & -3.0 & 272 \\
\hline $69 t$ & 808 & 4.00 & 1.0 & 2.231 & 16 & -13.3 & $\sim 0$ & 2.3 & -0.4 & 294 \\
\hline 64 & 805 & 3.74 & -3.2 & 2.234 & 22 & -14.9 & 11 & 4.9 & 1.2 & 310 \\
\hline 65 & 803 & 3.49 & -10.1 & 2.239 & 28 & -15.6 & 22 & 7.3 & 3.8 & 327 \\
\hline
\end{tabular}

Saturation temperature $T_{S}=822^{\circ} \mathrm{C}$

tComposition: Bi.0.73 Pro.s4 $\mathrm{Yb}_{2.23} \mathrm{Fe}_{9.66} \mathrm{Ga}_{1.02} \mathrm{O}_{12}$

The effect of Ga substitution on the birefringence was studied in YNdGaIG films [9]. Fig.4 shows the lattice mismatch dependence of $\Delta n_{f}$ and calculated $\Delta n_{f}{ }^{S}$ We found no remarkable difference between the cases of YNdIG and YNdGaIG films. This means that a combination of rare-earth ions on the dodecahedral site is important in generating the gaps between $\Delta n_{f}$ and $\Delta n_{f}{ }^{s}$.

The birefringence of various $B i$-substituted iron garnet films were also compared with the $\Delta n_{f} s$ calculated from the lattice mismatch[8 14]. Table 1 shows physical and guided-wave properties of BiPrYbGaIG films at $\lambda=1.15 \mu \mathrm{m}[10]$. In these films we find that $\Delta n_{f}$ is $I_{a r g e r}$ than $\Delta n_{f}{ }$ and the magnitude of the difference is the order of $10^{-4}$. The similar result has been observed in (110)oriented BiGdYbYAlIG films by Ageev et al. [25]. From the annealing experiments described later, we

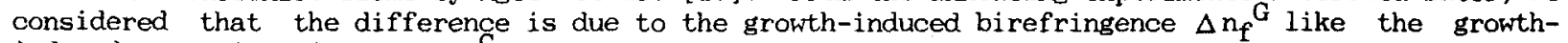
induced magnetic anisotropy $K_{U}$ in LPE garnet films.

Table 2 shows guided-wave properties of various iron garnet films at $\lambda=1.15 \mu \mathrm{m}[6,9,10,261$. It turns out that the garnet films are classified into three groups from the values of $\Delta \mathrm{n}_{\mathrm{f}} \mathrm{G}$ (1) $\Delta n_{f} G_{0}$ (YIG, YTbAIIG), (2) $\Delta n_{f} G_{<0}$ (YGdIG, YNdIG, YSmIG, YNdGaIG), and (3) $\Delta n_{f}>0$ (BiNdYGaIG, BiPrYbGaIG, BiGdLuGaIG).

This classification suggests that there is a certain correlation between $\Delta n_{f}{ }^{G}$ and $K_{u}{ }^{G}$ Because the $K_{u}{ }^{G}$ is known to arise when more than two rare-earth ions with different ionic radii occupy the inequivalent dodecahedral sites in the film growth process. Furtheremore, it is noted that $B i-s u b s t i t u t e d$ iron garnet films have large $K_{u} G$ in general, although its detaild mechanism is not made clear yet [3].

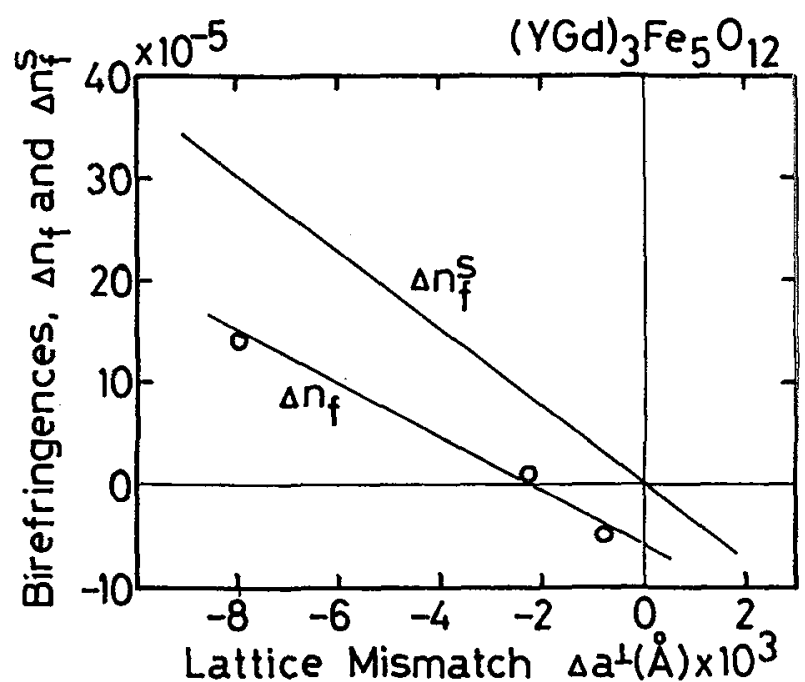

Fig. 3 Film birefringence $\Delta n_{f}$ and stress-induced birefringence $\Delta n_{f}$ in YGdIG films as a function of lattice mismatch $\Delta a^{\perp}[9]$.

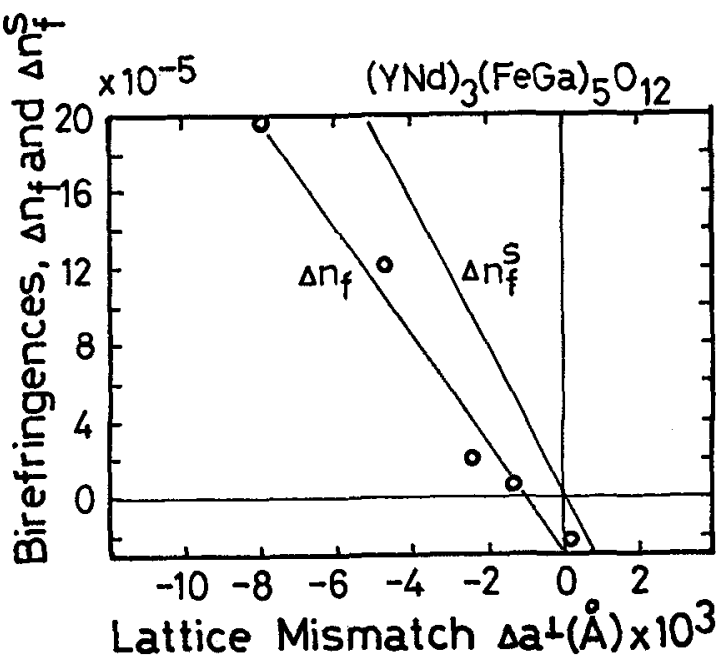

Fig.4 Film biref ringence $\Delta \mathrm{n}_{\mathrm{f}}$ and stress-induced birefringence $\Delta n_{f}$ in YNdGaIG films as a function of lattice mismatch $\Delta a \perp$ [9]. 
Table 2 Physical and guided-wave properties of iron garnet films at $\lambda=1.15 \mu \mathrm{m} . \quad \Delta n_{f}=\Delta n_{f}-\Delta n_{f}$ S : growth-induced birefringence.

\begin{tabular}{|c|c|c|c|c|c|c|c|c|c|}
\hline Material & $\begin{array}{c}d \\
(\mu \mathrm{m})\end{array}$ & $\begin{array}{l}\Delta a^{I} \\
(A)\end{array}$ & $\mathbf{n}_{\mathbf{f}}$ & $\begin{array}{c}K \\
(\mathrm{rad} / \mathrm{cm})\end{array}$ & $\begin{array}{c}\Delta \beta \\
(\mathrm{rad} / \mathrm{cm})\end{array}$ & $\Delta \mathbf{n}_{\mathbf{f}}$ & $\Delta n_{f} S$ & $\Delta n_{f}^{G}$ & Ref. \\
\hline & & $10^{-3}$ & & & & $10^{-1}$ & $10^{-4}$ & $10^{-4}$ & \\
\hline $\mathrm{Y}_{3} \mathrm{Fe}_{53} \mathrm{O}_{12}$ & 4.08 & 8.2 & 2.213 & 4.1 & -29.5 & -3.0 & -9.1 & 0.1 & 9 \\
\hline $\mathrm{Y}_{1.76} \mathrm{~Tb}_{1.2 \times} \mathrm{Pb}_{0.02} \mathrm{Fe}_{4.7} \mathrm{Al}_{0.20} \mathrm{O}_{12}$ & 7.6 & -1.1 & 2.19 & 6.7 & 1.8 & 0.3 & 0.4 & 0.1 & 6 \\
\hline $\mathrm{Y}_{2.78} \mathrm{Cd}_{0.22} \mathrm{Fe}_{8} \mathrm{O}_{12}$ & 4.77 & -2.2 & 2.210 & 5.0 & -7.0 & 0.1 & 0.9 & -0.8 & 26 \\
\hline $\mathrm{Y}_{2 . \mathrm{ns}} \mathrm{Nd}_{0.15} \mathrm{Fe}_{3} \mathrm{O}_{12}$ & 5.84 & 0.5 & 2.213 & 4.4 & -8.5 & -0.7 & 0.2 & -0.7 & 9 \\
\hline $\mathrm{Y}_{2.78} \mathrm{Sm}_{0.24} \mathrm{Fe}_{8} \mathrm{O}_{18}$ & 3.65 & -10.1 & 2.212 & 3.9 & 2.3 & 3.4 & 3.8 & -0.4 & 9 \\
\hline $\mathrm{Y}_{2.04} \mathrm{Nd}_{0.26} \mathrm{Fe}_{4.25} \mathrm{Ca2.75}_{0.72} \mathrm{O}_{12}$ & 3.80 & $-\mathbf{8 . 0}$ & 2.177 & 2.0 & -3.4 & 2.0 & 3.0 & -1.0 & 9 \\
\hline$(\mathrm{BiNdY})_{3}(\mathrm{FeCa})_{5} \mathrm{O}_{12}$ & 5.03 & -32.5 & 2.207 & -5.4 & $22.4 \uparrow$ & 15.0 & 12.3 & 2.7 & 9 \\
\hline $\mathrm{Bi}_{0.73} \mathrm{Pr}_{0.94} \mathrm{Yb}_{2.2 \mathrm{~s}} \mathrm{Fe}_{3.64} \mathrm{Ca}_{1.02} \mathrm{O}_{12}$ & 4.00 & 1.0 & 2.231 & -13.3 & $\mathbf{0}$ & 2.3 & -0.4 & 2.7 & 10 \\
\hline $\mathrm{Bi}_{0.65} \mathrm{Cd}_{0.97} \mathrm{Lu}_{1.81} \mathrm{Fe}_{3.96} \mathrm{Ca}_{0.71} \mathrm{O}_{12}$ & 4.13 & 5 & 2.244 & -10.1 & 0.5 & 2.2 & -2 & 4.2 & 26 \\
\hline
\end{tabular}

+ Mode order $m=2$.

\section{4-2 Annealing effects in Bi garnet films.}

We studied the effects of annealing on the residual birefringence in Bi garnet films $[8,11]$.

Fig. 5 shows the $\Delta n_{f}$ of as-grown films and annealed films as a function of $\Delta a^{\perp}$ for BiGdLuGaIG films grown by using a PbO- $\mathrm{B}_{2} \mathrm{O}_{3}-\mathrm{Bi}_{2} \mathrm{O}_{3}$ flux. The $\Delta \mathrm{n}_{\mathrm{f}}$ of annealed films are extrapolated values to infinite time deduced from the annealing time dependence at $1150^{\circ} \mathrm{C}$. It turns out that the values of $\Delta n_{f}$ in annealed films coincide with those of the stress-induced birefringence $\Delta n_{f} S$. That is, the difference between $\Delta n_{f}$ and $\Delta n_{f}$ disappears on annealing.

Fig.6 shows annealing time dependences of $\Delta n_{f}$, the uniaxial magnetic anisotropy constant $K$ and the saturation magnetization $4 \pi \mathrm{Ms}$ in BiGdLuGaIG films. As seen here, the annealing time dependence is almost the same for $\Delta n_{f}$ and $K_{u}$; the time constants for the changes of $\Delta n_{f}$ and $K_{u}$ are much the same at $1100 \sim 1150^{\circ} \mathrm{C}$. From these results we concluded that both $\Delta n_{f}{ }^{G}$ and $k_{u}{ }^{G}$ are caused by the specific microscopic structure formed during the growth process.

\section{4-3 Bi content dependence}

The $\mathrm{Bi}$-substitution of dodecahedral rare-earth ions in garnets affects their magnetic, optical and magneto-optical properties such as the Curie temperature $\mathrm{T}_{\mathrm{C}}$, uniaxial magnetic anisotropy constant $\mathrm{K}_{u}$, refractive index $\mathrm{n}_{f}$, and Faraday rotation $\theta_{F}$ [3]. Especially, since the growthinduced birefringence $\Delta n_{f}$. is thought to be related with the growth-induced uniaxial anisotropy constant $K_{u}{ }^{G}$, the $\mathrm{Bi}$ content dependence of these properties was studied for BiYGaIG and BiYAlIG films $[13,14]$.

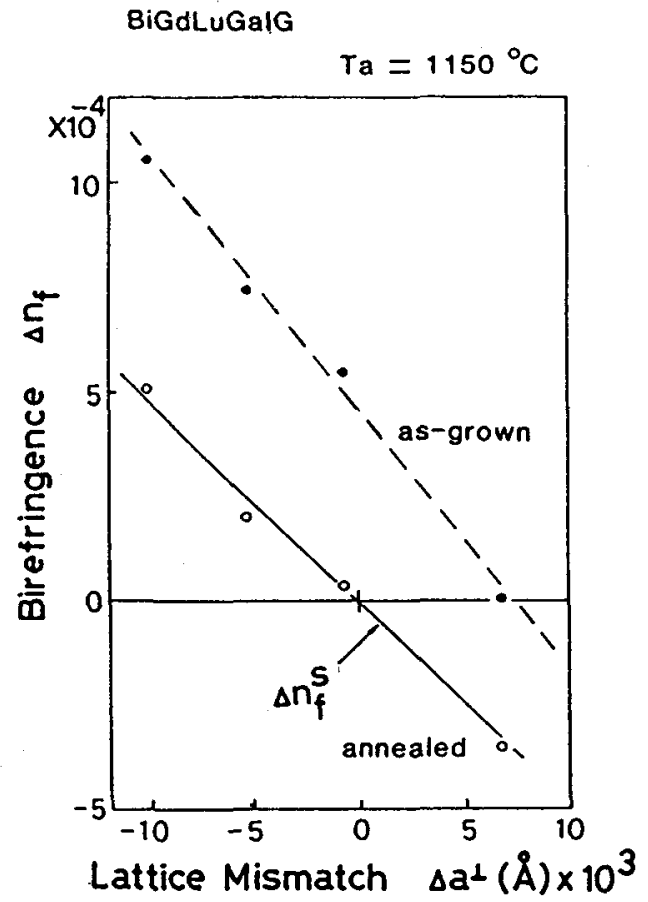

Fig.5 Film birefringence $\Delta n_{f}$ of as-grown $(\bullet)$ annealed $(0)$ films and stress-induced birefringence $\Delta \mathrm{n}_{\mathrm{f}} \mathrm{S}(\longrightarrow)$ as a function of lattice mismatch $\Delta \mathrm{a} \perp[11]$.

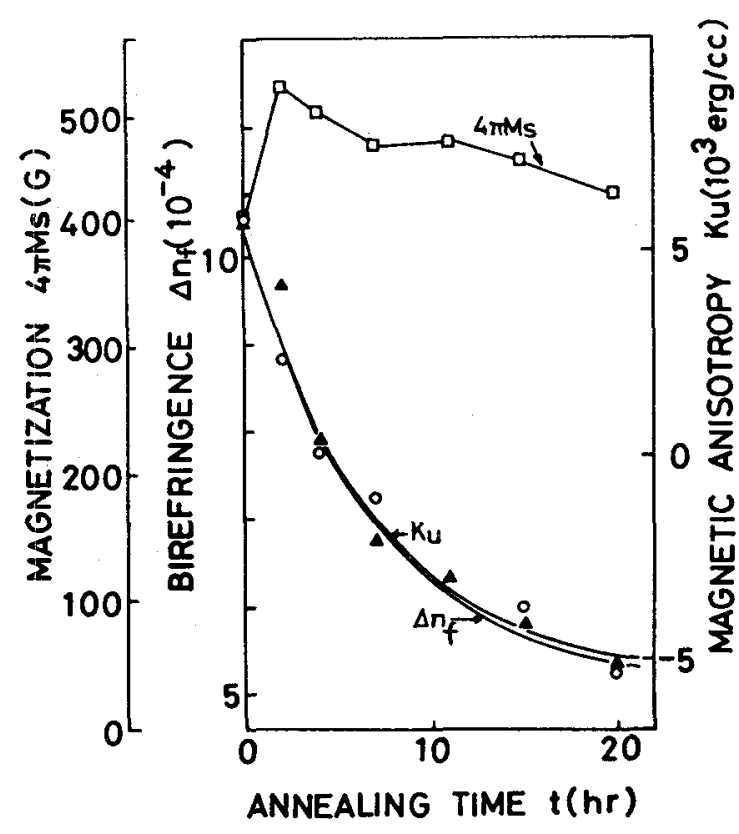

Fig.6 Film birefringence $\Delta \mathrm{n}_{\mathrm{f}}(0)$, uniaxial magnetic anisotropy constant $\mathrm{K}_{\mathrm{u}}(\boldsymbol{\Delta})$ and saturation magnetization $4 \pi \mathrm{Ms}(\square)$ as a function of annealing time. The annealing temperature $\mathrm{Ta}=1150{ }^{\circ} \mathrm{C}[11]$. 
Table 3 Physical properties of $\mathrm{Y}_{3-\mathrm{x}} \mathrm{Bi}_{\mathrm{x}} \mathrm{Fe}_{5-\mathrm{y}} \mathrm{M}_{\mathrm{y}} \mathrm{O}_{12}(\mathrm{M}=\mathrm{Ga}, \mathrm{Al})$ at $\lambda=1.15 \mu \mathrm{m}[13]$. Ha : magnetic anisotropy field, $\theta_{F}:$ Faraday rotation.

\begin{tabular}{|c|c|c|c|c|c|c|c|c|c|}
\hline & $\begin{array}{l}\text { CONTENT } \\
\text { (atoms/f.u.) }\end{array}$ & & $\begin{array}{c}d \\
(\mu \mathrm{m})\end{array}$ & $\begin{array}{l}\Delta a^{\perp} \\
(A)\end{array}$ & $\begin{array}{c}4 \times M_{6} \\
(G)\end{array}$ & $\begin{array}{c}H_{\mathbf{z}} \\
(\mathrm{Oe})\end{array}$ & $\begin{array}{c}\theta_{F} \\
(\operatorname{deg} / \mathrm{cm})\end{array}$ & $n_{f}$ & $\Delta n_{f}^{G}$ \\
\hline$x_{\mathrm{B} i}$ & $y_{0 k}$ & $y_{\mathrm{Al}}$ & & $10^{-3}$ & & & & & $10^{-4}$ \\
\hline 0 & 0 & 0 & 4.08 & 8.2 & 1,750 & - & 240 & 2. 213 & 0.1 \\
\hline 0.15 & 0.45 & 0 & 4. 91 & $<1.0$ & 942 & 280 & -63 & 2. 191 & $<0.5$ \\
\hline 0.24 & 0.77 & 0 & 3.51 & 3.4 & 339 & 409 & -211 & 2. 179 & 1.3 \\
\hline 0.32 & 0.95 & 0 & 6.43 & $<2.0$ & 89 & 2,170 & -258 & 2.171 & 2.4 \\
\hline 0.36 & 1.16 & 0 & 4. 20 & $<2.0$ & 16 & - & - & 2.160 & 3.5 \\
\hline 0.44 & o & 0.32 & 3.50 & $<2.0$ & 1,120 & 212 & -893 & 2.237 & 3.7 \\
\hline 0.61 & 0 & 0.47 & 4. 10 & $<2.0$ & 829 & 1,054 & $-1,280$ & 2. 241 & 8.1 \\
\hline 0.76 & 0 & 0.57 & 3.85 & $<2.0$ & 603 & 1,920 & $-1,390$ & 2. 242 & 12.6 \\
\hline 0.82 & 0 & 0.61 & 3.89 & $<2.0$ & 540 & 2,553 & - & 2.243 & 14.8 \\
\hline
\end{tabular}

Table 3 shows physical properties of BiYGaIG and BiYAlIG films [13]. From the atomic absorption analysis, the impurity content of $\mathrm{Pb}$ or $\mathrm{Pt}$ was estimated to be $0.01 \sim 0.02$ atoms/f.u..

Fig.7 shows $\mathrm{Bi}$ content dependence of $\mathrm{K}^{\mathrm{G}}[13]$. The $\mathrm{K}^{\mathrm{G}}$ was obtained from the value of $\mathrm{K}_{\mathrm{u}}$ by taking into account the stress-induced anisotropy constant $\mathrm{K}_{\mathrm{u}} \mathrm{S}$ and neglecting the relatively small crystalline anisotropy constant $\mathrm{K}_{1}$.

Fig. 8 shows $\mathrm{Bi}$ content dependence of refractive index $\mathrm{n}_{\mathrm{f}}$ at $\lambda=1.15 \mu \mathrm{m}$. In general, it is known that the $n_{f}$ increases with $\mathrm{Bi}$ content because of the optical red shift of the band gap. While, the $n_{f}$ decreases by the incorporation of nonmagnetic ions, $\mathrm{Ga}^{3+}$ and $\mathrm{Al}^{3+}$, into $\mathrm{Fe}^{3+}$ sites, because the absorption intensity of the Fe associated with charge transfer transitions and pair transitions decreases. The difference of $\mathrm{Bi}$ content dependence in $\mathrm{n}_{\mathrm{f}}$ between two series of films is explained by these factors.

Fig.9 shows Bi content dependence of $\Delta \mathrm{n}_{\mathrm{f}}^{\mathrm{G}}$ at $\lambda=1.15 \mu \mathrm{m}$ [13]. It turns out that the $\Delta \mathrm{n}_{\mathrm{f}}^{\mathrm{G}}$ increases monotonically with $\mathrm{Bi}$ content. The Bi content dependence of $\Delta \mathrm{n}_{\mathrm{f}} \mathrm{G}$ are different between BiYGaIG and BiYAlIG films as that of $K_{u} G$. However, the difference is not so large compared with the case of $n_{f}$. The contribution of Al ions to $\Delta n_{f} G$ seems to be small compared with that of $B i$ ions. The different $B i$ content dependence of $\Delta n_{f}$ between BiYGaIG and BiYAlIG films may be due to the difference in LPE-growth conditions such as melt composition and growth temperature. Recently, we have found that the magnitude of $\Delta n_{f}$ is dependent not only on the growth conditions but also on the lattice size of the substrate $[14]$.

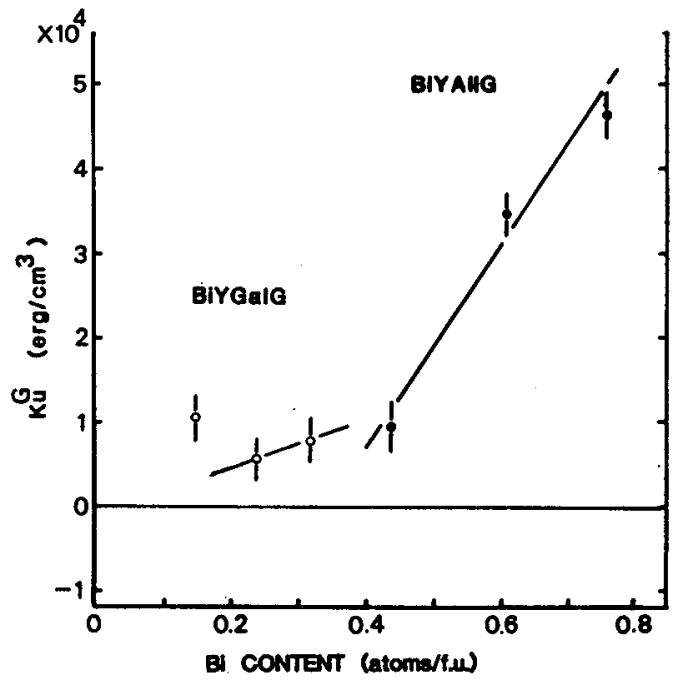

Fig.7 Growth-induced uniaxial magnetic anisotropy constant $K^{G}$ as a function of $B i$ content in BiYGaIG and BiYAIIG films [13].

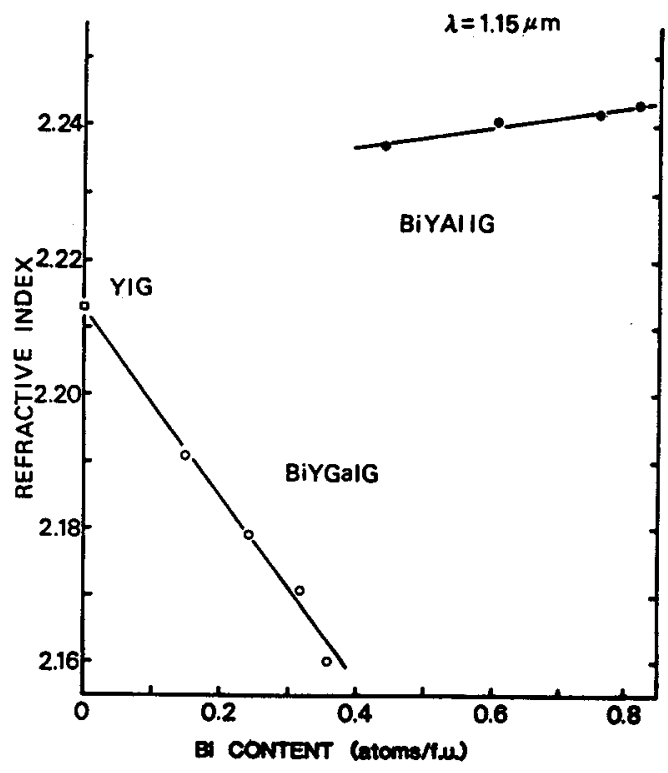

Fig.8 Refractive index of film $n_{f}$ as a function of Bi content in BiYGaIG and BiYAlIG films [13]. 


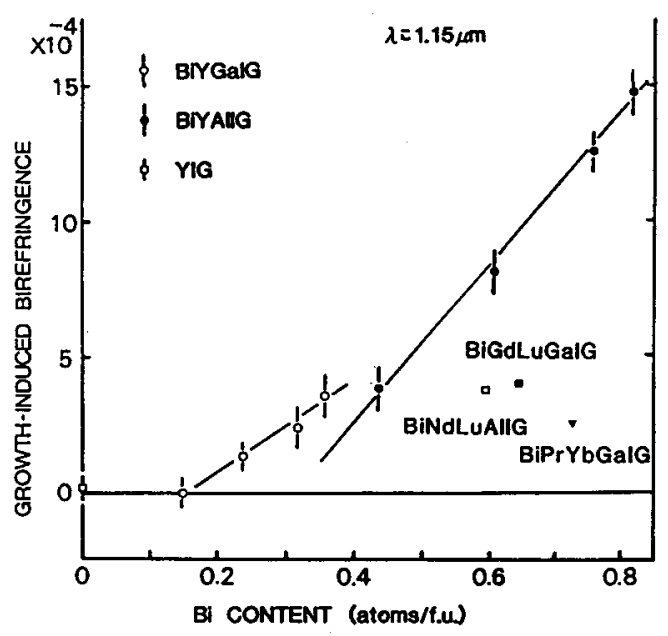

Fig. 9 Growth-induced birefringence $\Delta n_{f}^{G}$ as a function of $\mathrm{Bi}$ content in BiYGaIG and BiYAlIG films [13]

\section{SUMMARY}

Recent studies of growth-induced birefringence for LPE-grown iron garnet films, especially for $\mathrm{Bi}$-substituted garnet films were reviewed. The specific properties of $\Delta n_{f}{ }^{G}$ are as follows : (1) $\Delta n_{f}{ }$ is found in rare-earth iron garnet films containing more than two rare-earth ions and in $B i$ garnet films. (2) The sign of $\Delta n_{f} G$ is negative in rare-earth garnets and is positive in $B i$ garnets. (3) It is confirmed in $B i$ garnet films that the $\Delta n_{f}^{G}$ disappears by proper annealing and the annealing time constant is almost the same as that of $\mathrm{K}_{u} \mathrm{G}$. (4) The $\Delta n_{f} \mathrm{G}_{\text {increases with } \mathrm{Bi}}$ content, but the magnitude depends on various growth conditions.

From these results it is considered that the $\Delta \mathrm{n}_{\mathrm{f}} \mathrm{G}$ and $\mathrm{K}_{\mathrm{u}} \mathrm{G}$ originate in the same microscopic structure in garnets ; a non-equilibrium distribution of dodecahedral site ions and a consequent deformation of the microscopic structure. Especially, in the case of Bi garnets, the large ion radius and large electronic polarizability of $\mathrm{Bi}^{3+}$ may play an important role in generating the optical anisotropy.

From the applications of point of view, it is concluded that the positive sign and the magnitude of the $\Delta n_{f}$ in Bi-substituted garnet films are favorable for the phase matching in a planar waveguide.

The authors are much indebted to Y.Yokoyama, A.Murata, N.Takeda and T.Takahashi for sample preparation and characterization.

\section{REFERENCES}

[1] P.Paroli : Thin Solid Films, 114, 187 (1984).

[2] G.B.Scott and D.E.Lacklison : IEEE Trans. Magn. MAG-12, 292 (1976).

[3] P.Hansen and J.-P.Krumme : Thin Solid Films, 114, 69 (1984).

[4] G.Hepner, J.P.Castera and B.Desormiere : AIP Conf. Proc., 29, 659 (1975).

[5] P.G.van Engen : J. Appl. Phys., 49, 4660 (1978).

[6] L.Courtois, J.M.Desvignes, M.Torfeh, H.Le Gall and J.P.Castera : The Rare Earth in Modern Science and Technology (Plenum, 1978), p.487.

[7] N.Koshizuka, T.Okuda, Y.Yokoyama and K.Ando: J. Magn. Magn. Mat. 35, 167 (1983).

[8] K.Ando, N.Koshizuka, T.Okuda and Y.Yokoyama : Jpn. J. Appl. Phys., 22, L618 (1983).

[9] T.Okuda, N.Koshizuka, A.Murata, Y.Yokoyama and K.Ando : J. Appl. Phys., 55, 2176 (1984).

[10] N.Koshizuka, Y.Yokoyama, T.Okuda, K.Ando and A.Murata : J. Mag. Soc. Japan, 8, 129 (1984).

[11] K.Ando, N.Takeda, N.Koshizuka and T.Okuda : J. Appl. Phys., 57, 1277 (1985).

[12] A.Murata, N.Koshizuka, T.Okuda, K.Ando, A.Ito and K.Kawanishi : IEEE Trans. Magn., MAG-21, 1657 $(1985)$.

[13] N. Koshizuka, Y.Yokoyama, K.Ando and T.Okuda : J. Mag. Soc. Japan, 10, 155 (1986) [in Japanese]

[14] T.Takahashi, N.Koshizuka, T.Okuda, K.Ando, Y.Yokoyama and K.Hayashi : to be published in the Proc. Int. Symp. Magneto-Optics (Kyoto, 1987).

[15] P.K.Tien, R.L.Martin, R.Wolfe, R.C.LeCraw and S.L.Blank : Appl. Phys. Lett., 21, 394 (1972).

[16] S.Wang, M.Shah and J.D.Crow : IEEE J. Quantum Electron., QE-8, 212 (1972).

[17] S.Yamanoto, Y.Koyamada and T.Makimoto : J. Appl. Phys., 43, 6090 (1972).

[18] S.Yamamoto and T.Makimoto : J. Appl. Phys., 45, 882 (1974).

[19] J.P.Castera and G.Hepner : IEEE Trans. Magn. MAG-13, 1583 (1977).

[20] M.Torfeh, J.M.Desvigne, L.Courtois and H.Le Gall : J. Appl. Phys., 49, 1806 (1978).

[21] S.T.Kirsch, W.A.Biolsi, S.L.Blank, P.K.Tein, R.J.Martin, P.M.Bridenbaugh and P.Grabbe : J. Appl. Phys., 52(5), 3190 (1981).

[22] Y.Miyazaki : J. Mag. Soc. Japan, 6, 254 (1982) [in Japanese].

[23] C.S.Tsai, D.Young, W,Chen, L.Adkins, C.C.Lee and H.Glass : Appl. Phys, Lett., 47(7), 651 (1985).

[24] K.Ando, N.Takeda, T.Okuda and N.Koshizuka : J. Appl. Phys., 57, 718 (1985).

[25] A.N.Ageev, O.G.Rutkin, A.S.Trifonov, V.B.Kravtchenko, L.M.Filimonova, H.Le Gall and J.M.Desvignes : Rev. Phys. Appl., 20, 753 (1985).

[26] N.Koshizuka, A.Murata, K.Ando and T.Okuda : unpublished work. 